\title{
Epitope Characterization and Variable Region Sequence of F1-40, a High-Affinity Monoclonal Antibody to Botulinum Neurotoxin Type A (Hall Strain)
}

\author{
Miles C. Scotcher ${ }^{1}$, Jeffery A. McGarvey ${ }^{1}$, Eric A. Johnson ${ }^{2}$, Larry H. Stanker ${ }^{1 *}$ \\ 1 United States Department of Agriculture, Agricultural Research Service, Western Regional Research Center, Albany, California, United States of America, 2 Department of \\ Bacteriology, Food Research Institute, University of Wisconsin-Madison, Madison, Wisconsin, United States of America
}

\begin{abstract}
Background: Botulism, an often fatal neuroparalytic disease, is caused by botulinum neurotoxins (BoNT) which consist of a family of seven serotypes $(\mathrm{A}-\mathrm{H})$ produced by the anaerobic bacterium Clostridium botulinum. BoNT, considered the most potent biological toxin known, is a $150 \mathrm{kDa}$ protein consisting of a $100 \mathrm{kDa}$ heavy-chain $(\mathrm{Hc})$ and a $50 \mathrm{kDa}$ light-chain (Lc). F1-40 is a mouse-derived, IgG1 monoclonal antibody that binds the light chain of BoNT serotype A (BoNT/A) and is used in a sensitive immunoassay for toxin detection. We report the fine epitope mapping of F1-40 and the deduced amino acid sequence of the variable regions of the heavy and light chains of the antibody.

Methods and Findings: To characterize the binding epitope of F1-40, three complementary experimental approaches were selected. Firstly, recombinant peptide fragments of BoNT/A light-chain were used in Western blots to identify the epitope domains. Secondly, a peptide phage-display library was used to identify the specific amino acid sequences. Thirdly, the three-dimensional structure of BoNT/A was examined in silico, and the amino acid sequences determined from the phagedisplay studies were mapped onto the three-dimensional structure in order to visualize the epitope. F1-40 was found to bind a peptide fragment of BoNT/A, designated L1-3, which spans from T125 to L200. The motif QPDRS was identified by phage-display, and was mapped to a region within L1-3. When the three amino acids Q138, P139 and D140 were all mutated to glycine, binding of F1-40 to the recombinant BoNT/A light chain peptide was abolished. Q-138, P-139 and D-140 form a loop on the external surface of BoNT/A, exposed to solvent and accessible to F1-40 binding.
\end{abstract}

Conclusions: The epitope of F1-40 was localized to a single exposed loop ( $34, \beta 5)$ on the LC of BoNT. Furthermore amino acids Q138, P139 and D140 forming the tip of the loop appear critical for binding.

Citation: Scotcher MC, McGarvey JA, Johnson EA, Stanker LH (2009) Epitope Characterization and Variable Region Sequence of F1-40, a High-Affinity Monoclonal Antibody to Botulinum Neurotoxin Type A (Hall Strain). PLoS ONE 4(3): e4924. doi:10.1371/journal.pone.0004924

Editor: Frederic Andre Meunier, The University of Queensland, Australia

Received October 10, 2008; Accepted February 2, 2009; Published March 17, 2009

This is an open-access article distributed under the terms of the Creative Commons Public Domain declaration which stipulates that, once placed in the public domain, this work may be freely reproduced, distributed, transmitted, modified, built upon, or otherwise used by anyone for any lawful purpose.

Funding: This work was funded by the United States Department of Agriculture, Agricultural Research Service, CRIS project 5325-42000-043-00D. The funders had no role in study design, data collection and analysis, decision to publish, or preparation of the manuscript.

Competing Interests: The Unites States Department of Agriculture has applied for a patent covering the antibody described in this study.

* E-mail: Istanker@pw.usda.gov

\section{Introduction}

Botulinum neurotoxins (BoNTs), the causative agents of botulism, are the most potent naturally-occurring toxins known [1]. Seven different serotypes of BoNT, designated A to G, are produced predominantly by strains of Clostridium botulinum, but also by some strains of $C$. butyricum and C. baratii [2]. Each serotype is further divided into one or more subtypes based upon strain of origin, resulting in over 40 immunologically distinct BoNT types [3]. BoNT/A (subtype Al), derived from the Hall strain of $C$. botulinum (ATCG 3502), is possibly the most widely studied and best understood of the BoNTs, and is used in this study.

BoNT is released following bacterial lysis as a $900 \mathrm{kDa}$ complex in association with several non-toxic accessory proteins (NAPs) [4]. The BoNT/A holotoxin is comprised of a $100 \mathrm{kDa}$ heavy chain (Hc) and a $50 \mathrm{kDa}$ light chain (Lc), linked by a single disulphide bond. The Hc functions by binding nerve cells and facilitates the internalization of the Lc, a zinc endopeptidase that cleaves
SNARE (soluble N-ethylmaleimide sensitive factor attachment receptor) proteins. This action prevents the release of acetylcholine from the neuron into the neuromuscular junction, ultimately resulting in flaccid paralysis of the muscle [5,6]. The threedimensional structure of the BoNT/A holotoxin has been determined at $3.3 \AA$ resolution [7].

In mice, an $\mathrm{LD}_{50}$ of $10 \mathrm{pg}$ per organism was reported for BoNT/A when administered by inter-peritoneal injection [8]. Early studies estimated a minimum human lethal dose $\left(\mathrm{LD}_{100}\right)$ of $\mathrm{BoNT} / \mathrm{A}$ at $1 \mathrm{ng}$ per $\mathrm{kg}$ body mass when administered via interperitoneal injection [9], but it has since been recognized that the route of toxin exposure is critical in determining its lethality. For example, the human $\mathrm{LD}_{100}$ for BoNT/A is estimated at $1 \mu \mathrm{g} / \mathrm{kg}$ when administered orally, whereas via intravenous injection, the $\mathrm{LD}_{100}$ is 700 -fold lower [10,11]

Although outbreaks of foodborne BoNT poisoning are rare, their impact can be significant. In July 2007, four cases of foodborne botulism were linked to the consumption of hotdog chili 
sauce resulting in a recall of over 721,000 pounds of canned meat products in 49 states, at a projected cost to the manufacturer of \$35 million [12-14]. BoNT has been used in, or developed for biological weapons since the 1930's, and is still considered a credible threat to national security today, being classified as a Class A bioterrorism agent by the CDC $[15,16]$. It has been suggested that the most likely terrorist strategy would be to contaminate large batches of food or beverages with BoNT. One study described a mathematical model of where milk was intentionally adulterated [17].

These concerns demonstrate the need for tests to detect the presence of toxin in food. Such tests must be easily completed with a minimum amount of sample preparation, they should be sensitive, specific, and give accurate results in a timely manner. Despite the range of tests that have or are being developed, most require a skilled technician to prepare the sample and perform the test, and can take from several hours to four days to obtain results (for full reviews see $[18,19])$. We recently reported the development and partial characterization of four high-affinity monoclonal antibodies (mAbs), designated F1-2, F1-5, F1-40 and F2-43, to BoNT/A from the Hall strain of C. botulinum [20]. Two of these mAbs, F1-2 and F1-40, were used to develop a sensitive sandwichELISA with a detection limit of $2 \mathrm{pg} / \mathrm{mL}$, approximately 10 -fold more sensitive than the mouse bioassay. Since F1-40 is used in this sensitive ELISA, molecular characterization and a detailed understanding of its binding properties are highly desirable to develop improved assays and enhance the predictive value of this diagnostic test. For example, F1-40 exhibits high affinity binding to the light chain of BoNT/A1, but other serotypes are not detected [20]. Understanding the molecular basis of this specificity would improve our ability to incorporate F1-40 into multianalyte assays able to detect more than a single serotype. In addition to development of improved diagnostics, mapping antibody epitopes onto the toxin is extending our understanding of the mechanisms by which antibodies, individually or in combinations, neutralize toxin $[21,22]$. Preliminary data indicates that F1-40, by itself or as a component of a multi-antibody mixture is effective in protecting mice from BoNT/A [23]. Thus, characterization of the F1-40 epitope may help elucidate the mechanism by which it neutralizes the toxin. Furthermore, since F1-40, in a mouse model, was able to rescue animals after intoxication, cloning and sequencing the antibody heavy and light chain variable regions represents the first step in further engineering of this antibody.

In this article, we report the identification of the F1-40 epitope on the light chain of BoNT/A using three complementary experimental approaches. Firstly, antibody binding to recombinant peptide fragments of BoNT/A light-chain was investigated. Secondly, a peptide phage-display library was used to identify amino acid sequences that were bound by F1-40. Thirdly the three-dimensional structure of BoNT/A was examined in silico, and the ligands determined from the phage-display studies were mapped onto the three-dimensional structure in order to visualize a putative epitope. Mutagenesis studies were used to confirm the identified epitope. In an effort to further characterize mAb F1-40, the variable regions gene sequences of the heavy and light chains were determined, and the amino acid sequences of the complementary determining regions deduced.

\section{Methods}

\section{Plasmid construction}

Commercial enzymes (Phusion High-Fidelity DNA Polymerase, BamHI, XhoI, T4 polynucleotide kinase [ $3^{\prime}$ phosphatase minus], T4 DNA ligase [New England BioLabs Inc., Bethesda, MD]) were used according to the manufacturer's recommendation. Primers used were purchased from Integrated DNA Technologies (Coralville, IA) and are shown in Table 1 . Plasmid construction and manipulation was performed in Escherichia coli TOP10 cells (Invitrogen, Carlsbad, CA) grown aerobically in Luria-Bertani (LB) medium at $37^{\circ} \mathrm{C}$ supplemented with $100 \mu \mathrm{g} / \mathrm{mL}$ ampicillin [24]. Plasmids or DNA were purified using the QuickClean 5M range of kits (GenScript Corp., Piscataway, NJ). All automated DNA sequencing was performed using the Big Dye Terminator Version 3.1 and XTerminator reagents, and a 3730 DNA Analyzer (Applied Biosystems, Foster City, CA).

Total genomic DNA from Clostridium botulinum (Strain ATCC3502) was used as a template to amplify the fragments of the light chain (Lc, L1, L2) using the primers indicated (see Figure 1 and Table 1). Stop codons (TAA) were introduced when not present within the genomic DNA of the cloned region. All subsequent BoNT/A DNA fragments were cloned into plasmid pCR4-TOPO (Invitrogen) to allow sequencing using primers $\mathrm{M} 13 \mathrm{~F}$ and M13R. The pCR4-derived plasmids were then digested using BamHI and XhoI, the BoNT/A fragment was purified and ligated into BamHI- and XhoI-digested pGS-2la (Genscript) to yield the correspondently named pGS plasmid (e.g. pGS-L1 for fragment L1). All pGS-21a-derived plasmids were sequenced using primer pGS-F and pGS-R, to confirm the correct integration of the BoNT/A fragment into the vector. The BamHI and XhoI cloning sites of pGS-21a are located downstream of glutathione-Stransferase (GST), under the control of the T7 promoter.

Plasmids pGS-L1-1 through pGS-L1-4 were constructed by PCR using plasmid pGS-L1 as a template. Primers were used to amplify outwards from the Ll region, thus eliminating internal pieces of L1 (see Figure 1). The PCR product was gel purified, treated with $\mathrm{T} 4$ polynucleotide kinase then self-ligated to form an intact plasmid. The mutant plasmids pGS-Lc- $\Delta$, pGS-Lc-QPD and pGS-Lc-RS (see Table 2) were constructed by PCR in an identical manner, using plasmid pGS-Lc as a template, and the primers shown in Table 1.

\section{Expression and purification of GST-fusion proteins}

All pGS-21a-derived plasmids were transformed into E. coli BL21-CodonPlus (DE3)-RIPL cells (Stratagene, La Jolla, CA), and grown aerobically on $\mathrm{LB}$ agar at $37^{\circ} \mathrm{C}$ supplemented with $100 \mu \mathrm{g} /$ $\mathrm{mL}$ ampicillin and $75 \mu \mathrm{g} / \mathrm{mL}$ streptomycin. Single colonies were grown overnight in LB containing the same antibiotics and $0.5 \%$ glucose, to minimize uninduced expression of the fusion protein [25]. An inoculum of $6 \mathrm{~mL}$ was added to $600 \mathrm{~mL} 2 \mathrm{YT}$ medium ( $1 \%$ tryptone, $1.6 \%$ yeast extract, $0.5 \% \mathrm{NaCl}$ ) containing the same antibiotics and glucose, and the culture was grown aerobically at $30^{\circ} \mathrm{C}$ with $200 \mathrm{rpm}$ agitation to an $\mathrm{OD}_{600}$ of $\sim 0.6$. Expression was induced with $1 \mathrm{mM}$ IPTG (isopropyl B-D-1 thiogalactopyranoside), the culture was grown overnight at $18^{\circ} \mathrm{C}$ and cells were harvested by centrifugation at $10000 \times \mathrm{g}$ for $10 \mathrm{~min}$.

The cell pellet was suspended in $10 \mathrm{~mL}$ lysis solution (PBS [10 mM phosphate buffer, $138 \mathrm{mM} \mathrm{NaCl}, 2.7 \mathrm{mM} \mathrm{KCl}, \mathrm{pH}$ 7.4], $1 \mathrm{mM}$ PMSF (phenylmethanesulphonylfluoride), $0.2 \mathrm{mg} / \mathrm{mL}$ lysozyme, $1 \times$ CelLytic-B Cell Lysis Reagent, $0.5 \mu \mathrm{L}$ Benzonase Nuclease [Sigma-Aldrich Inc., St. Louis, MO]) and incubated at $37^{\circ} \mathrm{C}$ for $10 \mathrm{~min}$ with $200 \mathrm{rpm}$ agitation, then placed immediately on ice. The lysate was clarified by centrifugation at $12800 \times g$ for $15 \mathrm{~min}$, then loaded directly onto a column of High-Affinity GST Resin (1 mL bed volume; Genscript) that had been equilibrate with 20 bed volumes ice-cold PBS containing $1 \mathrm{mM}$ PMSF. The column was washed with 30 bed volumes ice-cold PBS-PMSF and then eluted with 15 bed volumes of elution buffer $(10 \mathrm{mM}$ reduced glutathione, $50 \mathrm{mM}$ Tris, $\mathrm{pH} 8.0$ ) in fractions of $1 \mathrm{~mL}$. 
Table 1. Primers.

\begin{tabular}{|c|c|c|}
\hline Primer & Sequence & Constructs \\
\hline LcF & GGATCCATGCCATTTGTTAATAAACAATTTAATTATAAAG & Lc, L1 \\
\hline LCR & CTCGAGTTATTTAGAAGTTATTATCCCTCTTACAC & Lc, L2 \\
\hline L1R & CTCGAGTTAAAGTGACTCCTCAAAACCAAATG & L1 \\
\hline L2F & GGATCCGAAGTTGATACAAATCCTCTTTTAG & L2 \\
\hline GS-L & GGATCCGATATCAGCCATGGCC & L1-3, L1-4 \\
\hline GS-R & TAACTCGAGCACCACCACCAC & $\mathrm{L} 1-1, \mathrm{~L} 1-2$ \\
\hline L1b & TTCTGGTGGTGGATTTAAATCTCCTTC & L1-1 \\
\hline L1c & GCAAAACAAGTTCCAGTTTCATATTATGATTC & L1-4 \\
\hline L1d & ATCTATTGTACTTCCACCCCAAAATGG & L1-2 \\
\hline L1e & ACAGAATTAAAAGTTATTGATACTAATTGTATTAATGTG & L1-3 \\
\hline $\mathrm{Lc}-\Delta \mathrm{L}$ & ATTAGTATCAATAACTTTTAATTCTGT & Lc- $\Delta$ \\
\hline Lc- $\Delta \mathrm{R}$ & CTTAATCTAGTAATAATAGGACCCT & Lc- $\Delta$ \\
\hline Lc-QPDL & ACCACCTATCACATTAATACAATTAGTATCAAT & Lc-QPD \\
\hline LC-QPDR & GGTGGTAGTTATAGATCAGAA & LC-QPD \\
\hline Lc-RSL & ACCATAACTACCATCTGGTTGTATCA & Lc-RS \\
\hline Lc-RSR & GGAGAAGAACTTAATCTAGTAATAATA & Lc-RS \\
\hline L-chainR & TCTAGAACTGGATGGTGGGAGATGGA & F1-40 L-chain cloning \\
\hline H-chainR & TCTAGAACCTCCACACACAGGAACCAGTGGATAGAC & F1-40 H-chain cloning \\
\hline L-chainF3 & GATATCCACCATGGAGTCACAGACTCAGGTCTTTGTA & F1-40 L-chain cloning \\
\hline H-chainF3 & GATATCCACCATGGCTGTCTTGGGGCTGCTCTTCT & F1-40 H-chain cloning \\
\hline M13F & GTAAAACGACGGCCAG & seq. pCR4 plasmids \\
\hline M13R & CAGGAAACAGCTATGAC & seq. pCR4 plasmids \\
\hline pGS-F & CAAATTGATAAGTACTTGAAATCC & seq. PGS-21a plasmids \\
\hline pGS-R & GCTAGTTATTGCTCAGAGG & seq. pGS-21a plasmids \\
\hline
\end{tabular}

Sites for restriction enzymes BamHI (GGATCC), Xhol (CTCGAG), Xbal (TCTAGA) and EcoRV (GATATC) are shown underlined. Stop codons are shown in bold, either in the $5^{\prime}$ to $3^{\prime}$ (TAA) or $3^{\prime}$ to $5^{\prime}$ (TTA) orientation. The third column indicates which peptide fragments each primer was used to construct, or where the primer was used to clone the heavy and light chain variable regions of F1-40. Primers used only for sequencing are indicated by the abbreviation "seq."

doi:10.1371/journal.pone.0004924.t001

\section{Electrophoresis and Western blots}

All gel electrophoresis equipment, buffers, gels and nitrocellulose membranes were purchased from PAGEgel (San Diego, CA). Samples of $40 \mu \mathrm{L}(25 \mu \mathrm{L}$ protein sample, $10 \mu \mathrm{L} 4 \times$ gel buffer, $4 \mu \mathrm{L} 1 \mathrm{M}$ dithiothreitol) were heated for $10 \mathrm{~min}$ at $70^{\circ} \mathrm{C}$, then loaded onto a $10 \%$ gel and separated by electrophoresis at $175 \mathrm{~V}$ (constant) for $80 \mathrm{~min}$. For protein visualization, gels were silver stained using the Silver Express kit (Invitrogen) according to manufacturer's instructions. For Western blotting, the gel was subjected to horizontal blotting onto a nitrocellulose membrane using a PAGEgel transfer cell (180 mA constant, $100 \mathrm{~min})$. Membranes were incubated in blocking buffer $(1 \times$ PBS-T $[$ PBS plus $0.05 \%$ Tween-20], 3\% non-fat dried milk) for $1 \mathrm{hr}$ at room temperature with gentle agitation. Membranes were incubated for $1 \mathrm{hr}$ at $37^{\circ} \mathrm{C}$ with gentle agitation in primary antibody (F140 or a rabbit polyclonal to Clostridium botulinum A toxoid \# 20641 [Abcam Inc., Cambridge, MA]) diluted $5 \mu \mathrm{g} / \mathrm{mL}$ in $10 \mathrm{~mL}$ of blocking buffer. Membranes were washed 4 times in PBS-T. Membranes were incubated for $1 \mathrm{hr}$ at room temperature with gentle agitation in secondary antibody diluted 1:5000 in blocking buffer. Peroxidase-conjugated, goat anti-mouse IgG \#A4416 was used to detect F1-40; peroxidase-conjugated, goat anti-rabbit IgG \#A6154 was used to detect the polyclonal antibody (Sigma-Aldrich). Membranes were washed 4 times in PBS-T, then SuperSignal West Dura Extended Duration Substrate (Pierce, Rockford, IL) was added according to manufacturer's instructions. Membranes were visualized using a Fluorchem SP unit (Alpha Innotech Corp., San Leandro, CA).

\section{Competition ELISA}

Black 96-well plates were coated with the Lc-GST fusion peptide by adding $100 \mu \mathrm{L}$ per well of a $4 \mu \mathrm{g} / \mathrm{mL}$ solution of $\mathrm{Lc}$ peptide in $0.05 \mathrm{M}$ carbonate buffer $(\mathrm{pH}$ 9.6) and incubating overnight at $4^{\circ} \mathrm{C}$. Plates were then blocked by adding $300 \mu \mathrm{L}$ blocking buffer $(1 \times$ PBS-T [PBS plus $0.05 \%$ Tween-20], $3 \%$ nonfat dried milk) to each well and incubating for $1 \mathrm{hr}$ at room temperature. Wells were filled with $100 \mu \mathrm{L}$ blocking buffer containing decreasing concentrations $(120,60,30,15,7.5,3.75$, $1.88,0.94$, and $0 \mu \mathrm{g} / \mathrm{mL}$ ) of a fusion peptide in solution (Lc, Lc- $\Delta$, Lc-QPD or Lc-RS) and then $100 \mu \mathrm{L}$ of a $5 \mu \mathrm{g} / \mathrm{mL}$ solution of F140 was immediately added to each well. Plates were incubated overnight at $4^{\circ} \mathrm{C}$ with gentle agitation, then washed 12 times in PBS-T. Next, $200 \mu \mathrm{L}$ of peroxidase-conjugated goat anti-mouse IgG \#A4416 (Sigma-Aldrich) diluted 1:5000 in blocking buffer was added to each well and the plate incubated for $1 \mathrm{hr}$ at room temperature with gentle agitation. Plates were washed 12 times in 


\section{Light chain}
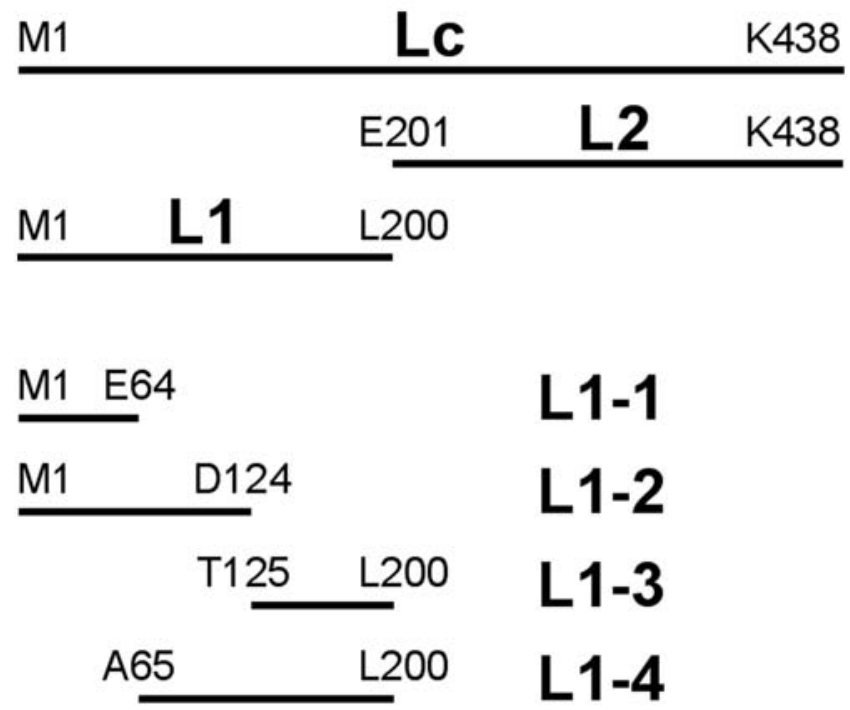

Scale

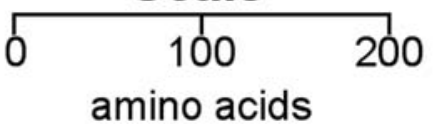

Figure 1. Peptide fragments of BoNT/A light chain. Diagram is drawn to scale to facilitate size and location comparison between peptide fragments. Peptide fragments were expressed as fusions to GST at the $\mathrm{N}$-terminal. $\mathrm{N}$ - and $\mathrm{C}$-terminal amino acids of each peptide fragment are indicated.

doi:10.1371/journal.pone.0004924.g001

PBS-T. Wells were filled with $100 \mu \mathrm{L}$ of SuperSignal ELISA Femto Maximum Sensitivity Substrate (Pierce) and incubated for $3 \mathrm{~min}$ at room temperature with gentle agitation. Luminescent counts were recorded using a Wallac Victor 2 Multilabel Counter (PerkinElmer Inc., Waltham, MA). The percentage inhibition of binding was calculated by the formula $\left(1-\mathrm{B} / \mathrm{B}_{0}\right) \times 100$, where $\mathrm{B}=$ luminescent counts at each concentration of fusion peptide in solution and $\mathrm{B}_{0}=$ luminescent counts at $0 \mu \mathrm{g} / \mathrm{mL}$ fusion peptide in solution.

\section{Phage Display}

The Ph.D.-C7C Phage Display Peptide Library Kit (New England BioLabs) was used to pan against $60 \mathrm{~mm}$ polystyrene dishes coated with antibody F1-40, to identify peptide ligands to F1-40, according to manufacturer's instructions. The M13 phage displays a randomized amino acid heptamer between two cysteine residues on the $\mathrm{pIII}$ minor coat protein. The cysteine residues form a disulphide bond, resulting in the heptamer being presented as constrained loop. Four series of panning and three phage amplification stages were carried out, prior to the growth and sequencing of individual plaques of the phage. A total of twelve clonal plaques from the $4^{\text {th }}$ pan were picked for sequencing.

\section{Cloning and sequencing of antibody variable regions}

The method used here for the cloning and sequencing of variable regions for antibody heavy and light chains is based upon
Table 2. Mutations made in the putative F1-40 epitope region on plasmid $\mathrm{pGS}-\mathrm{Lc}$.

\begin{tabular}{ll}
\hline & \\
\hline Vector & Light chain, from T-125 to Q-162 \\
\hline pGS-Lc & TELKV I DTNC I NV I QPDGSYRSEELNLVIIGPSADIIQ \\
pGS-LC- $\Delta$ & TELKV I DTN - - - - - - - - LN LV I I GPSAD I I Q \\
pGS-LC-QPD & TELKV I DTNC I NV I GGGGSYRSEELNLVIIGPSADIIQ \\
pGS-LC-RS & TELKV I DTNC I NV I QPDGSY GGEELNLVIIGPSADIIQ \\
\hline
\end{tabular}

All four vectors shown above harbored and expressed the entire BoNT/A light chain (P-1 to K-438), but only the mutated region (T-125 to Q-162) is shown here. All other amino acids within the light chain were not mutated. Dashes indicate that amino acids have been deleted. Single amino acid mutations are indicated in bold.

doi:10.1371/journal.pone.0004924.t002

the method described fully in Current Protocols in Immunology [26]. Significant changes from this methodology are described here.

Hybridoma cells for F1-40 production were grown as previously described [20]. Culture volumes of $5 \mathrm{~mL}$ were centrifuged at $12000 \times \mathrm{g}$ for $1 \mathrm{~min}$ to collect the cells. mRNA was purified from the cells using Trizol Reagent (Invitrogen), according to manufacturer's instructions. cDNA was transcribed into mRNA using primers $\mathrm{H}$-chainR and $\mathrm{L}$-chainR, and AMV reverse transcriptase according to manufacturer's instructions (Promega, Madison, WI) The cDNA was amplified by PCR using Phusion DNA polymerase and the appropriate pairs of primers $(\mathrm{H}$-chain $\mathrm{R}$ and F3; L-chain R and F3). The cDNA was gel purified, then treated using the MasterTaq Kit (Eppendorf North America, Westbury, NY) according to manufacturer's instructions to add Aoverhangs to the DNA. The cDNA was then cloned into plasmid pCR4-TOPO, and transformed into TOP10 cells. Six individual colonies of $E$. coli harboring pCR4-H-chain and pCR4-L-chain were picked, grown and prepared for sequencing as described earlier.

\section{Computer tools}

All DNA and amino acid sequence analysis was carried out using the tools of the Biology Workbench, Version 3.2 (http:// workbench.sdsc.edu San Diego Supercomputer Center, University of California, San Diego, CA). The three-dimensional diagrams of BoNT/A were generated using the crystal structure of BoNT/A [7]) manipulated with the MBT Protein Workshop (RCSB Protein Data Bank, www.rcsb.org/pdb).

\section{Results}

Binding of $\mathrm{F} 1-40$ to peptide fragments of the BoNT/A light chain

Seven recombinant peptide fragments of BoNT/A light chain (Lc, L1, L2, L1-1, L1-2, L1-3, L1-4) are shown in Figure 1. These recombinant peptides were expressed as fusions to glutathione-Stransferase (GST). The relative molecular weights (rMW) of these recombinant peptide-GST proteins, observed by SDS-PAGE and silver staining, are shown in Figure 2, panels A and C. In each case, the rMW corresponds to that predicted for the recombinant peptide-GST protein. Results from Western blot analyses are shown in panels B and D. Clearly, F1-40 bound to the light-chain fragment (Lc) and to subfragment L1, but binding to L2 was not detected (Figure 2, Panel B). The L1 peptide fragment corresponds to amino acids M1 to L200. This region was further subdivided by generating four additional recombinant peptide fragments L1-1 

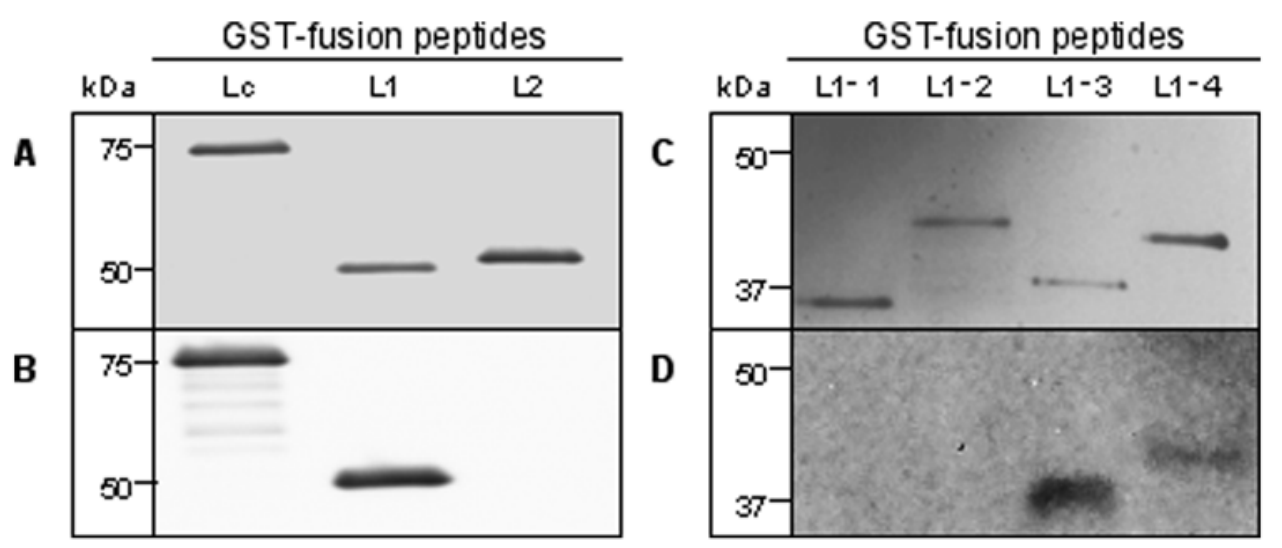

Figure 2. Binding of F1-40 to peptide fragments of BoNT/A light chain. A and C, Light chain fragment-GST fusion peptides separated by gel electrophoresis and stained with Silver Stain. B and D, Corresponding Western Blots using F1-40 for primary detection. Fusion peptide size is indicated in $\mathrm{kDa}$.

doi:10.1371/journal.pone.0004924.g002

through L1-4 (Figure 1). In Western blotting experiments, F1-40 was observed to bind two of these recombinant peptide fragments, L1-3 and L1-4 (Figure 2, Panel D). In contrast, binding to L1-1 and L1-2 was not detected. These results, particularly antibody binding to L1-3, suggest that the epitope for F1-40 resides between amino acids T125 and L200.

\section{Fine epitope maping of F1-40 using heptamer peptide ligands identified by phage display}

In an effort to further localize the epitope for F1-40, binding to a random phage display library of heptamer peptides was evaluated. Following four rounds of panning, twelve clonal plaques picked for sequencing. One plaque failed to yield sequence, and the remaining eleven sequences of the heptamer peptide ligands for F1-40 are shown, with their frequency of occurrence, in Table 3. The most common motif, SSAFYPK, found in eight of the eleven plaques sequenced, did not readily map to a putative epitope region on the L1-3 peptide fragment, or to any other region on the light chain of BoNT/A and most probably represents a mimotope. In contrast, the motif QPDRS, common to the remaining three heptamer sequences, when mapped onto the three-dimensional structure of BoNT/A [7] corresponded to the turn (Q139, P140, D141) between B4-B5 and the first two amino acids (R144, S145) of B5. While R144 and S145 are separated from amino acids QPD (the very tip of the

Table 3. Amino acid sequences identified using phage display against F1-40.

\begin{tabular}{ll}
\hline Heptamer sequence & Frequency \\
\hline S S A F Y P K & 8 \\
T R Q P D R S & 1 \\
$T$ L Q P D R S & 1 \\
S L Q P D R S & 1 \\
\hline $\begin{array}{l}\text { The sequences of the heptamer peptide ligands to mAb F1-40 were identified } \\
\text { using the Ph.D.-C7C Phage Display Peptide Library Kit (New England BioLabs } \\
\text { Inc.) to pan against mAb F1-40. Twelve clonal phage plaques were sequenced, } \\
\text { one failed to yield sequence, and the frequency of the heptamer sequences } \\
\text { observed is shown. } \\
\text { doi:10.1371/journal.pone.0004924.t003 }\end{array}$
\end{tabular}

loop) they are brought into close proximity to each other by the tertiary structure of the toxin (See Figure 3).

Binding of F1-40 to mutants of the Lc peptide fragment

We developed mutant recombinant Lc peptides carrying specific amino acid deletions or substitutions within the region identified via phage display (Table 2). The region spanning from C134 to E148 (B4-15) forms a looped structure, the tip of which corresponds to Q139-P140-D141. The entire loop was deleted from the recombinant peptide Lc to form mutant peptide Lc- $\Delta$. In mutant peptide Lc-QPD the amino acids Q139, P140 and D141 were all mutated to glycine. In mutant peptide Lc-RS the amino acids R145 and S146 were both mutated to glycine. Panel A in Figure 4 shows a silver stained gel of the Lc peptide fragment and the three mutant peptides. As a control, a polyclonal anti-BoNT/ A toxoid antiserum was used in a Western blot, which clearly bound the Lc peptide and all three mutant peptides (Panel B). Binding of F1-40 to these mutant peptides is shown by Western blot in Panel C. F1-40 bound the wild-type Lc peptide and the mutated peptide Lc-RS, but binding to mutated peptides Lc- $\Delta$ and to Lc-QPD was not detected. These data demonstrate that the mutation of the Q139, P140, D141 motif to GGG decreases F1-40 binding to a level which was not detected by Western blotting.

\section{Competition ELISA}

Competition ELISA assays were performed to better quantify the differences of antibody binding to the mutant peptides observed in the above Western blotting experiments. Results from these experiments are shown in Figure 5. Peptide Lc was the most effective at competing binding of F1-40 to immobilized Lc, with $50 \%$ inhibition achieved at a concentration of $\sim 3.5 \mu \mathrm{g} / \mathrm{mL}$. Mutant peptide Lc-RS was the next most effective competitor resulting in $50 \%$ inhibition of control activity at $\sim 24 \mu \mathrm{g} / \mathrm{mL}$, roughly 7 -fold higher than that observed for the recombinant Lc peptide. Finally, mutants Lc- $\Delta$ and Lc-QPD did not compete for F1-40 binding under these conditions, even at the highest concentration of peptide used $(60 \mu \mathrm{g} / \mathrm{mL})$.

\section{F1-40 heavy and light chain variable region sequences}

The cDNA sequences of the heavy and light chain variable regions for F1-40 are shown in Figure 6. The nucleotide sequence of the entire cloned regions is shown, with the corresponding amino acids shown from the start of the leader peptide to the end 


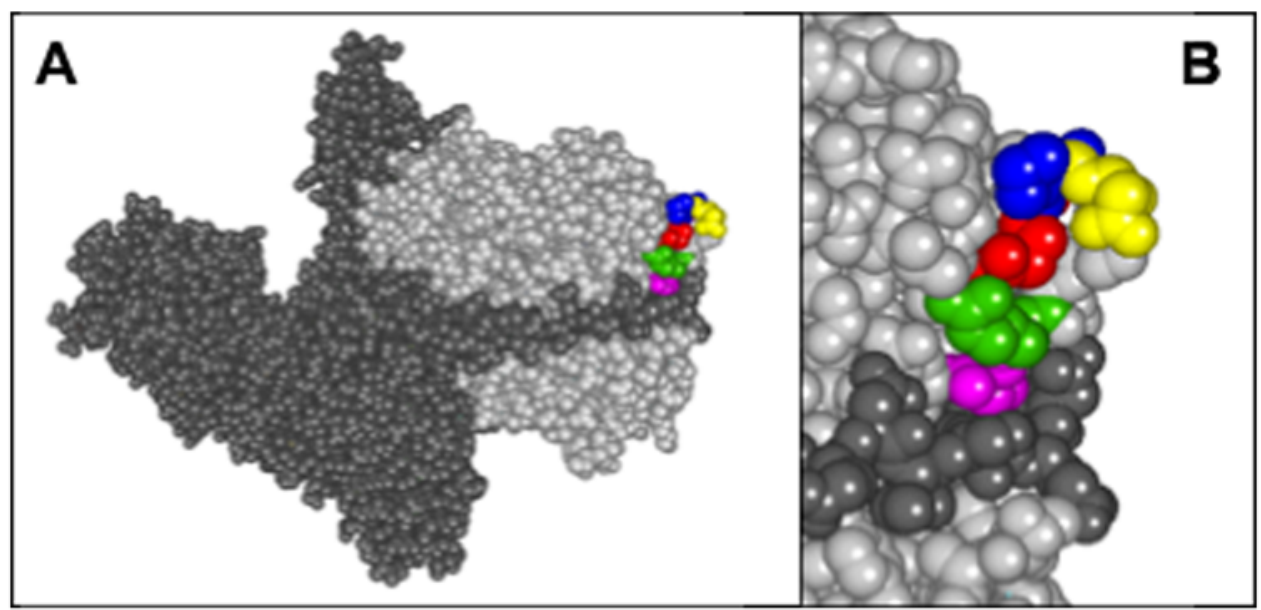

Figure 3. Location of the QPDRS motif within the BoNT/A holotoxin. A. Space-filled diagram of the three-dimensional model of the complete BoNT/A holotoxin showing the heavy (dark gray) and light (light gray) chains. QPDRS motif is the colored region within the light chain. B. Enlarged view of Q139 (red), P140 (blue), D141 (yellow), R145 (green) and S146 (pink) [7].

doi:10.1371/journal.pone.0004924.g003

of the fourth framework region of each chain. The leader sequences, framework regions, complementarity determining regions (CDRs) and J-regions were identified by inspecting the alignment of the F1-40 heavy and light chains to other antibody sequences [26-30]. The nucleotide sequence reported was identical across the six individual colonies of $E$. coli harboring either pCR4-L-chain or pCR4-H-chain that were analyzed. EMBL Nucleotide Sequence Database accession numbers for these sequences are FM177889 and FM177890, for the light and heavy chains, respectively.

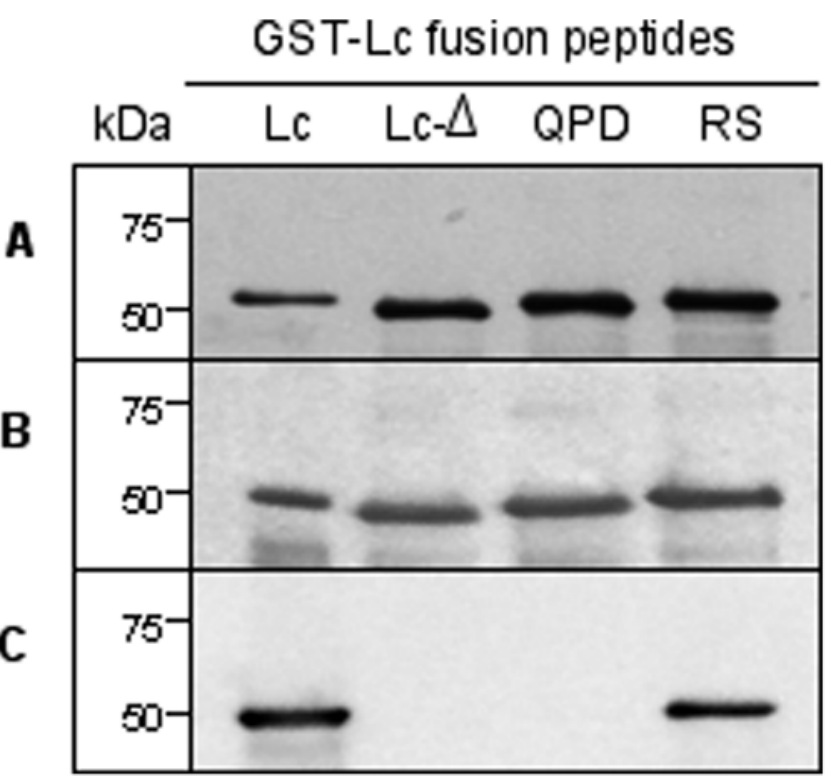

Figure 4. Binding of F1-40 to the Lc peptide fragment mutants. A., Lc peptide fragment mutants separated by gel electrophoresis and stained with Silver Stain. B, Western Blot using rabbit polyclonal to Clostridium botulinum A toxoid \# 20641 (Abcam Inc.) for primary detection. C, Western Blot using F1-40 for primary detection. Peptide size is indicated in $\mathrm{kDa}$.

doi:10.1371/journal.pone.0004924.g004

\section{Discussion}

The epitope of mAb F1-40 was mapped to the exposed loop located between B4-B5 on the light chain of BoNT/A (Figure 3). The amino acids QPDRS, identified by phage display experiments mapped to this region, Q139, P140, and D141 form the tip of the loop and R145 and S146 are brought into close proximity by the tertiary structure of BoNT (Figure 3).

Previous studies in our laboratory [20] demonstrated that mAb F1-40 bound the light chain domain (Lc) of BoNT. To more clearly define the binding epitope, the Lc domain of BoNT/A as well as smaller fragments were cloned into E. coli and expressed as GST fusion proteins (Figure 2). Western blotting experiments measuring antibody binding to these recombinant peptide fragments of toxin Lc demonstrated that the epitope for F1-40

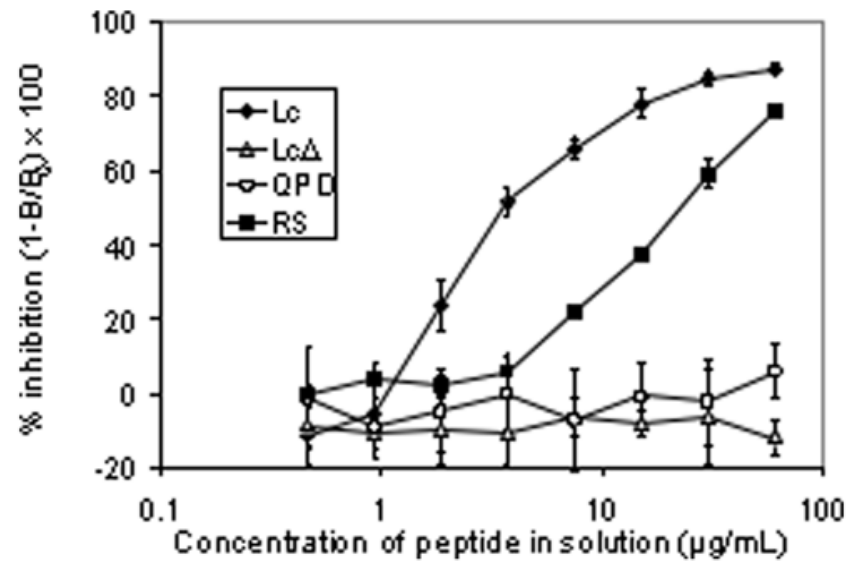

Figure 5. Competition ELISA for F1-40 by Lc peptide fragment mutants in solution. The percent inhibition of binding of $m A b$ F1-40 to immobilized LC peptide fragment on a 96-well plate by various peptide fragments in solution ( $\bullet$ Lc; $\triangle \mathrm{Lc}-\Delta ; \bigcirc \mathrm{Lc}-\mathrm{QPD} ; \mathrm{LC}-\mathrm{RS}$ ) was calculated by the formula $\left(1-B / B_{0}\right) \times 100$, where $B=$ luminescent counts at each concentration of fusion peptide in solution, and $B_{0}=$ luminescent counts at $0 \mu \mathrm{g} / \mathrm{mL}$ fusion peptide in solution. Data is presented \pm 1 standard error, $\mathrm{n}=4$.

doi:10.1371/journal.pone.0004924.g005 


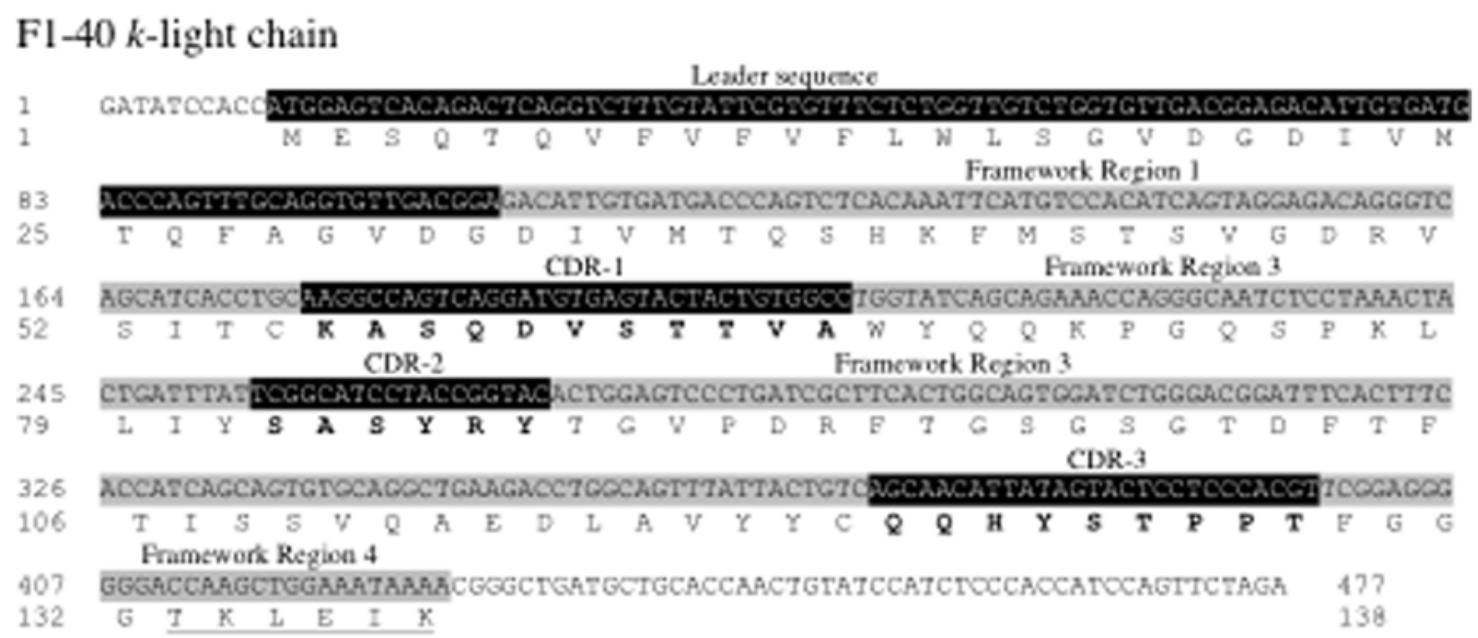

\section{Fl-40 heavy chain}

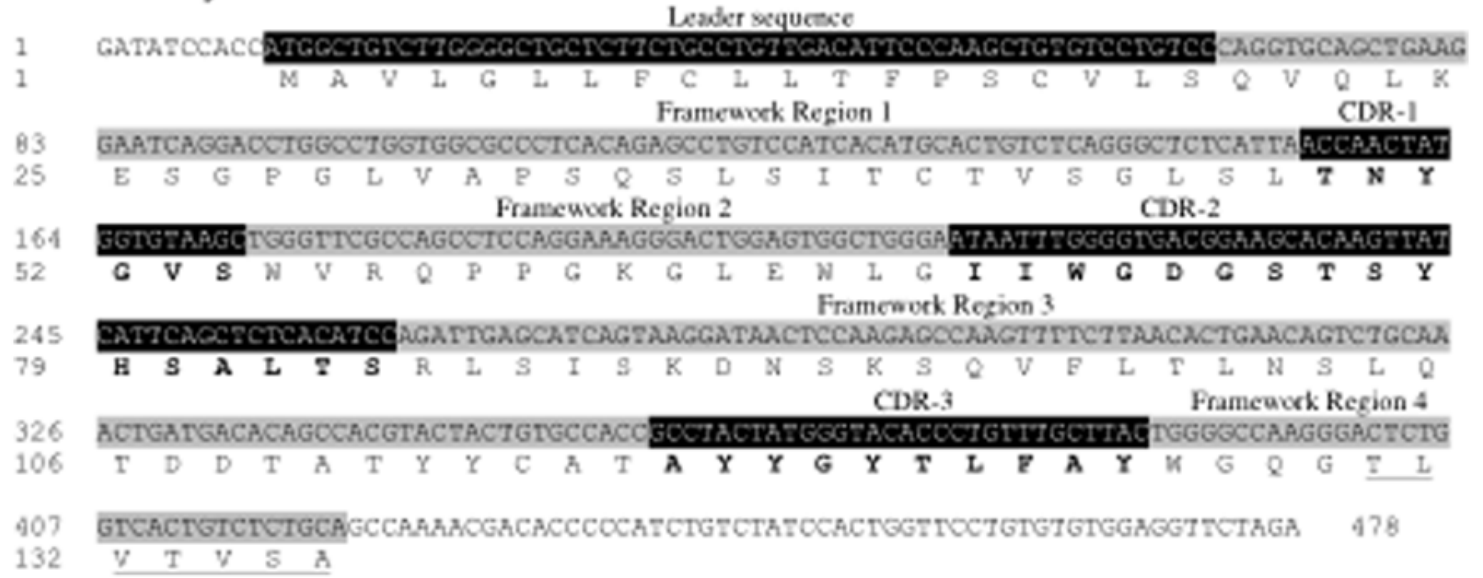

Figure 6. Nucleotide and amino acid sequences for the variable regions of F1-40 $\boldsymbol{k}$-light and heavy chains. The nucleotide sequences for the entire cloned regions are shown, with the deduced amino acids from the leader sequence to the end of the fourth framework region. The leader sequence and three complementarity determining regions (CDRs) are shown highlighted in black, with the amino acids of the CDRs shown in bold. The four framework regions are shown highlighted in light gray, with the amino acids of the J-regions of both light and heavy chains shown underlined, within the fourth framework region. EMBL online Accession \#s FM177889 \& FM177890.

doi:10.1371/journal.pone.0004924.g006

was contained within a 76 amino acids region from T125 to L200. Fine epitope mapping by analysis of binding sequences identified in phage display experiments suggested that a peptide ligand containing the amino acid motif QPDRS might constitute part or all of the F1-40 epitope. When these amino acids were mapped onto the three-dimensional crystal structure of BoNT/A [7], they formed a compact, almost continuous sequence corresponding to an external loop $(34,85)$ region of the molecule (Figure 3). Amino acids Q139, P140, and D141 form the tip of the loop and are separated from residues R145 and S146 by three intervening amino acids. However, the BoNT/A tertiary structure brings these amino acids R145 and S146 into close proximity of Q139, P140, and D141. Replacement of R145 and S146 with glycine residues had no obvious effect on F1-40 binding as illustrated by Western blot, but competition ELISA experiments revealed a 7-fold decrease in the relative affinity of mutant Lc-RS for F1-40 compared to the recombinant Lc peptide. These data suggest that under the conditions of SDS-PAGE, binding is not visibly altered by mutating R145 and S146. However, the competiton ELISA data demonstrated that R145 and S146 do contribute in part to the binding of F1-40 to the light chain of BoNT/A, under the solution conditions of competition ELISA. Mutation of Q139, P140 and D141 to glycine residues or eliminating the entire loop (mutant Lc $\Delta$ ) abolished binding of F1-40 to the Lc peptide in both the competition ELISA and Western blotting experiments, indicating that the QPD triad is necessary for the binding of F140 to the light chain of BoNT/A.

We have previously shown that binding of F1-40 to BoNT serotypes B through $\mathrm{G}$ is undetectable by ELISA [20]. All three amino acids, Q139, P140, D141, forming the turn of the exposed loop (B4-15) are exposed to solvent (Figure 3). Analysis of the amino acid sequence and three-dimensional structure of the light chain of BoNTs B through $G$ reveals that although a loop is formed in a similar position on the light chain in all other BoNT types (except BoNT/C - there is no crystal structure available), none possess the QPD motif in its entirety [31-35]. It is highly probable that the inability of F1-40 to bind the other serotypes of BoNT is due to the absence of a complete QPD motif. Interestingly, BoNT/G possesses Q144 and P145 (corresponding to Q139 and P140) but a glycine not aspartate residue at position 
146 (corresponding to D141), yet it remains unable to bind F1-40, leading to speculation about the possible role that D141 might play in determining the specificity of F1-40 to BoNT/A [20,34].

Using a series of overlapping synthetic peptides Dolimbek et al. [36] successfully mapped continuous regions of BoNT/B recognized by antibodies derived from human, horse and mouse sera. Such experiments can identify immunodominant areas of a protein and they have often been used to identify antibody binding epitopes. However, this approach only detects linear epitopes and overlooks complex, conformational epitopes. Using phage display libraries expressing BoNT Hc gene fragments, the epitopes of two Hc-specific anti-BoNT antibodies were identified [37]. Levy et al. [26] reported the effective use of a randomly mutated BoNT/A heavy chain library, displayed on the surface of yeast cells, to identify single amino acids important in the epitopes of anti-BoNT/A monoclonal antibodies. In the absence of such libraries for the BoNT/A light chain, we have nonetheless been able to characterize the epitope of F1-40 to a similarly high resolution. We expect our experimental approach to increase the likelihood of identifying epitopes of other antibodies in the future, and to facilitate their characterization at the single amino acid level.

Although the phage display analysis yielded the sequence QPDRS, it also provided the anomalous motif SSAFYPK in eight of the eleven sequenced plaques. Unlike the sequence QPDRS, this second sequence could not be mapped onto the light chain of BoNT/A, and it probably represents a mimotope of the F1-40 epitope. Mimotopes are commonly identified by phage display, and this faculty has been widely exploited to generate potential peptide vaccine candidates [22,38-39]. When using phage display to search for an epitope, multiple mimotopes that bind a monoclonal antibody can be selected, and it is necessary to examine every mimotope to identify an epitope region $[40,41]$.

\section{References}

1. Lamanna C (1959) The most poisonous poison. Science 130: 763-772.

2. Hatheway CL (1990) Toxigenic clostridia. Clin Microbiol Rev 3(1): 66-98.

3. Smith TJ, Lou J, Geren IN, Forsyth CM, Tsai R, et al. (2005) Sequence variation within botulinum neurotoxin serotypes impacts antibody binding and neutralization. Infection and immunity 73(9): 5450-5457.

4. Niwa K, Koyama K, Inoue S, Suzuki T, Hasegawa K, et al. (2007) Role of nontoxic components of serotype $\mathrm{D}$ botulinum toxin complex in permeation through a Caco-2 cell monolayer, a model for intestinal epithelium. FEMS Immunol Med Microbiol 49(3): 346-352.

5. Montecucco C, Schiavo G (1993) Tetanus and botulism neurotoxins: a new group of zinc proteases. Trends Biochem Sci 18(9): 324-327.

6. Montecucco C, Schiavo G (1994) Mechanism of action of tetanus and botulinum neurotoxins. Mol Microbiol 13(1): 1-8.

7. Lacy DB, Tepp W, Cohen AC, DasGupta BR, Stevens RC (1998) Crystal structure of botulinum neurotoxin type A and implications for toxicity. Nat Struct Biol 5(10): 898-902.

8. Wu HC, Yeh CT, Huang YL, Tarn LJ, Lung CC (2001) Characterization of neutralizing antibodies and identification of neutralizing epitope mimics on the Clostridium botulinum neurotoxin type A. Appl Environ Microbiol 67(7): 3201-3207.

9. Gill DM (1982) Bacterial toxins: a table of lethal amounts. Microbiol Rev 46(1): 86-94.

10. Brin MF (1997) Botulinum toxin: chemistry, pharmacology, toxicity, and immunology. Muscle Nerve Suppl 6: S146-168.

11. Sobel J (2005) Botulism. Clin Infect Dis 41(8): 1167-1173.

12. CDC (2007) Botulism associated with commercially canned chili sauce-Texas and Indiana, July 2007. MMWR Morb Mortal Wkly Rep 56(30): 767-769.

13. Gibb G (2007) Castleberry's Foods: A Modern-Day Botulism Scare. Available: http://www.lawyersandsettlements.com/articles/01266/castleberry-cannedmeat-recall.html.

14. Reynolds G (2007) Castleberry's recall could cost $\$ 35 \mathrm{~m}$. Available: http://www. foodproductiondaily-usa.com/news/ng.asp?id = 79051-castleberry-s-contaminationrecall.

15. Zilinskas RA (1997) Iraq's biological weapons. The past as future? Jama 278(5): 418-424.

16. Arnon SS, Schechter R, Inglesby TV, Henderson DA, Bartlett JG, et al. (2001) Botulinum toxin as a biological weapon: medical and public health management. JAMA 85(8): 1059-1070.
Supporting evidence defining an epitope, such as the Lc peptide mutagenesis data presented in this study, must be included in the analysis before assigning an epitope to an antibody. The assignment of an epitope motif to F1-40 including Q139, P140, D141, R145 and S146 is consistent with the BoNT serotype A specificity of F1-40.

Sequence analysis of the cDNA derived from the mRNA coding for the heavy and light chains of F1-40 reveals features that are typical of mouse monoclonals antibodies $[28,30,42]$. On the $k$-light chain, the section GVDGDIVMTQ from G29 to Q38 is a repeat of a preceding section from G17 to Q26, and forms the junction between the leader sequence and first framework region of the mature light chain. This repeat is not always present in $k$-light chains. The J-region of the heavy chain, TLVTVSA, is type 3 and the J-region of the $k$-light chain, TKLEIK, is type 2 [27].

In conclusion, we have localized the epitope of anti-BoNT/A $\mathrm{mAb}$ F1-40 to the exposed loop between B4-35 regions. Both the our phage display and mutant-binding data suggest that amino acids Q139, P140, and D141, located at the tip of this loop, are critical for antibody binding. In addition, we report the cDNA and deduced amino acid sequences the variable and J-regions of the antibody's heavy and light chains. The epitope assignment to a specific loop not only provides important insight into the nature of the interaction between F1-40 and BoNT/A, but also is highly useful for the future development of F1-40 as an integral component of a test for BoNT/A contamination of food.

\section{Author Contributions}

Conceived and designed the experiments: MCS LHS. Performed the experiments: MCS. Analyzed the data: MCS JAM LHS. Contributed reagents/materials/analysis tools: JAM EAJ. Wrote the paper: MCS. Extensive editorial review: LHS

17. Wein LM, Liu Y (2005) Analyzing a bioterror attack on the food supply: the case of botulinum toxin in milk. Proceedings of the National Academy of Sciences of the United States of America 102(28): 9984-9989.

18. Sharma SK, Whiting RC (2005) Methods for detection of Clostridium botulinum toxin in foods. J Food Prot 68(6): 1256-1263.

19. Lindstrom M, Korkeala H (2006) Laboratory diagnostics of botulism. Clin Microbiol Rev 19(2): 298-314.

20. Stanker LH, Merrill P, Scotcher MC, Cheng LW (2008) Development and partial characterization of high-affinity monoclonal antibodies for botulinum toxin type A and their use in analysis of milk by sandwich ELISA. Journal of immunological methods 336(1): 1-8.

21. Levy R, Forsyth CM, LaPorte SL, Geren IN, Smith LA, et al. (2007) Fine and domain-level epitope mapping of botulinum neurotoxin type A neutralizing antibodies by yeast surface display. Journal of molecular biology 365(1): 196-210.

22. Adekar SP, Takahashi T, Jones RM, Al-Saleem FH, Ancharski DM, et al. (2008) Neutralization of botulinum neurotoxin by a human monoclonal antibody specific for the catalytic light chain. PLoS ONE 3(8): e3023.

23. Cheng L, Stanker LH, Henderson TD, Scotcher MC (2008) Antibody protection of mice from botulinum intoxication; $45^{\text {th }}$ Interagency Botulinum Research Coordinating Committee Meeting; Philadelphia, PA. pp VA-8.

24. Miller JH (1972) Experiments in molecular genetics. Cold Spring Harbor, N.Y.: Cold Spring Harbor Laboratory Press.

25. Hengen PN (1996) Methods and reagents. Purification of GST fusion proteins. Trends Biochem Sci 21(10): 400-401.

26. Morrison S (2002) Cloning, expression and modification of antibody V regions. Current Protocols in Immunology. pp 2.12.11 to 12.12.17.

27. Wood DL, Coleclough G (1984) Different joining region J elements of the murine kappa immunoglobulin light chain locus are used at markedly different frequencies. Proceedings of the National Academy of Sciences of the United States of America 81(15): 4756-4760.

28. Recinos A 3rd, Silvey KJ, Ow DJ, Jensen RH, Stanker LH (1994) Sequences of cDNAs encoding immunoglobulin heavy- and light-chain variable regions from two anti-dioxin monoclonal antibodies. Gene 149(2): 385-386.

29. Recinos A 3rd, Silvey KJ, Ow DJ, Jensen RH, Stanker LH (1995) Sequences of cDNAs encoding immunoglobulin heavy- and light-chain variable regions from two anti-dioxin monoclonal antibodies [Gene 149 (1994) 385-386]. Gene 158(2): 311-312. 
30. Livesay DR, Subramaniam S (2004) Conserved sequence and structure association motifs in antibody-protein and antibody-hapten complexes. Protein Eng Des Sel 17(5): 463-472.

31. Swaminathan S, Eswaramoorthy S (2000) Structural analysis of the catyalytic and binding sites of Clostridium botulinum neurotoxin B. Nat Struct Biol 7(8): 693-699.

32. Agarwal R, Eswaramoorthy S, Kumaran D, Binz T, Swaminathan S (2004) Structural analysis of botulinum neurotoxin type $\mathrm{E}$ catalytic domain and its mutant Glu212 $\rightarrow$ Gln reveals the pivotal role of the Glu212 carboxylate in the catalytic pathway. Biochemistry 43(21): 6637-6644.

33. Agarwal R, Binz T, Swaminathan S (2005) Structural analysis of botulinum neurotoxin serotype $\mathrm{F}$ light chain: implications on substrate binding and inhibitor design. Biochemistry 44(35): 11758-11765.

34. Arndt JW, Yu W, Bi F, Stevens RC (2005) Crystal structure of botulinum neurotoxin type $\mathrm{G}$ light chain: serotype divergence in substrate recognition. Biochemistry 44(28): 9574-9580.

35. Arndt JW, Chai Q Christian T, Stevens RC (2006) Structure of botulinum neurotoxin type D light chain at 1.65 A resolution: repercussions for VAMP-2 substrate specificity. Biochemistry 45(10): 3255-3262.
36. Dolimbek BZ, Steward LE, Aoki KR, Atassi MZ (2008) Immune recognition of botulinum neurotoxin $\mathrm{B}$ : antibody-binding regions on the heavy chain of the toxin. Mol Immunol 45(4): 910-924.

37. Mullaney BP, Pallavicini MG, Marks JD (2001) Epitope mapping of neutralizing botulinum neurotoxin A antibodies by phage display. Infection and immunity 69(10): 6511-6514.

38. Hill HR, Stockley PG (1996) Phage presentation. Mol Microbiol 20(4): 685-692.

39. Manoutcharian K, Gevorkian G, Cano A, Almagro JC (2001) Phage displayed biomolecules as preventive and therapeutic agents. Curr Pharm Biotechnol 2(3): 217-223.

40. Li Y, Ning YS, Wang YD, Hong YH, Luo J, et al. (2007) Production of mouse monoclonal antibodies against Helicobacter pylori Lpp20 and mapping the antigenic epitope by phage display library. Journal of immunological methods 325(1-2): 1-8.

41. Li Y, Ning YS, Wang YD, Luo J, Wang W, et al. (2008) Production of mouse monoclonal antibodies against Helicobacter pylori Catalase and mapping the antigenic epitope by phage display library. Vaccine 26(9): 1263-1269.

42. Kabat EA, Wu TT, Reid-Miller M, Perry H, Gottesman K (1987) Sequences of Proteins of Immunological Interest, Fourth Edition: US Department of Health and Human Services, Public Health Service, National Institute of Health, Bethesda, MD. 